\title{
Annuaire des Enseignements en Andrologie
}

\author{
Présentation
}

En 2001, le Conseil d'Administration de la SALF a mandaté la Commission Enseignement de la société, afin qu'elle recense les enseignements en rapport avec l'Andrologie, effectifs sur notre territoire. En France, l'Andrologie ne figure pas au titre des spécialités médicales reconnues, bien que nombre de cliniciens se considèrent andrologues.... De fait, la SALF regroupe des praticiens de spécialités diverses, médicales, chirurgicales et biologiques. C'est à l'évidence cette pluralité qui fait toute la richesse de notre société, conduisant à l'ouverture et à la confrontation de connaissances et de pratiques différentes, mais dont le but consensuel est la prise en charge de l'homme, au sens masculin.

Les enseignements ayant trait à l'Andrologie témoignent eux aussi de cette très grande diversité dans l'abord de notre discipline, tant dans le niveau des diplômes obtenus que dans le contenu enseigné. Le travail de recensement a été initié sur le principe d'une approche la plus large possible, afin de répondre aux attentes du plus grand nombre de personnes intéressées par le domaine de l'Andrologie et souhaitant améliorer leurs connaissances, quel que soit leur niveau préalable.

La Commission Enseignement de la SALF a donc

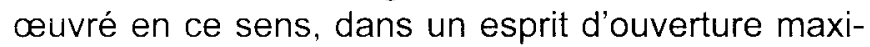
male ; elle est en mesure de communiquer le recensement ci-dessous, qui a le mérite d'exister, mais n'est certes pas exhaustif et comporte, très certainement, des inexactitudes et des oublis. C'est pourquoi, la Commission Enseignement sollicite l'indulgence des responsables d'enseignements en Andrologie qui ne figurent pas sur cette liste et les remercie de bien vouloir l'en informer. II en est de même pour toute erreur repérée dans un libellé d'enseignement, le nom ou les coordonnées d'un responsable.
Le projet de la Commission est en effet de mettre à jour régulièrement ce listing et de le tenir à la disposition de tous. Cependant, au delà des éventuels changements de responsable(s) ou d'appellation des enseignements actuels, il est évident que l'application de la réforme $L M D$ va probablement remanier profondément le paysage de l'enseignement de l'Andrologie et la participation de tous est indispensable pour que nous puissions actualiser efficacement cette liste.

De façon à diffuser le plus possible des informations pratiques concernant ces différents enseignements, une mise à jour de cet Annuaire des Enseignements en Andrologie sera publié chaque année dans le numéro de Mars de la revue Andrologie.

Par avance, la Commission Enseignement de la SALF vous remercie de votre compréhension et de votre coopération.

Martine Albert et la Commission Enseignement de la SALF

\section{Pour toute correspondance concernant l'Annuai- re des Enseignements en Andrologie :}

Pr Marie-Roberte Guichaoua Laboratoire de BDR, Hôpital Conception 147 Bd Baille, 13385 Marseille cedex 5 mguichaoua@ap-hm.fr 


\section{Enseignements en Andrologie}

Liste établie par la Commission Enseignement de la SALF

Pour toute correspondance :

Pr Marie-Roberte GUICHAOUA

Laboratoire de BDR,

Hôpital Conception, 147 Boulevard Baille,

13385 MARSEILLE CEDEX 5

mguichaoua@ap-hm.fr 


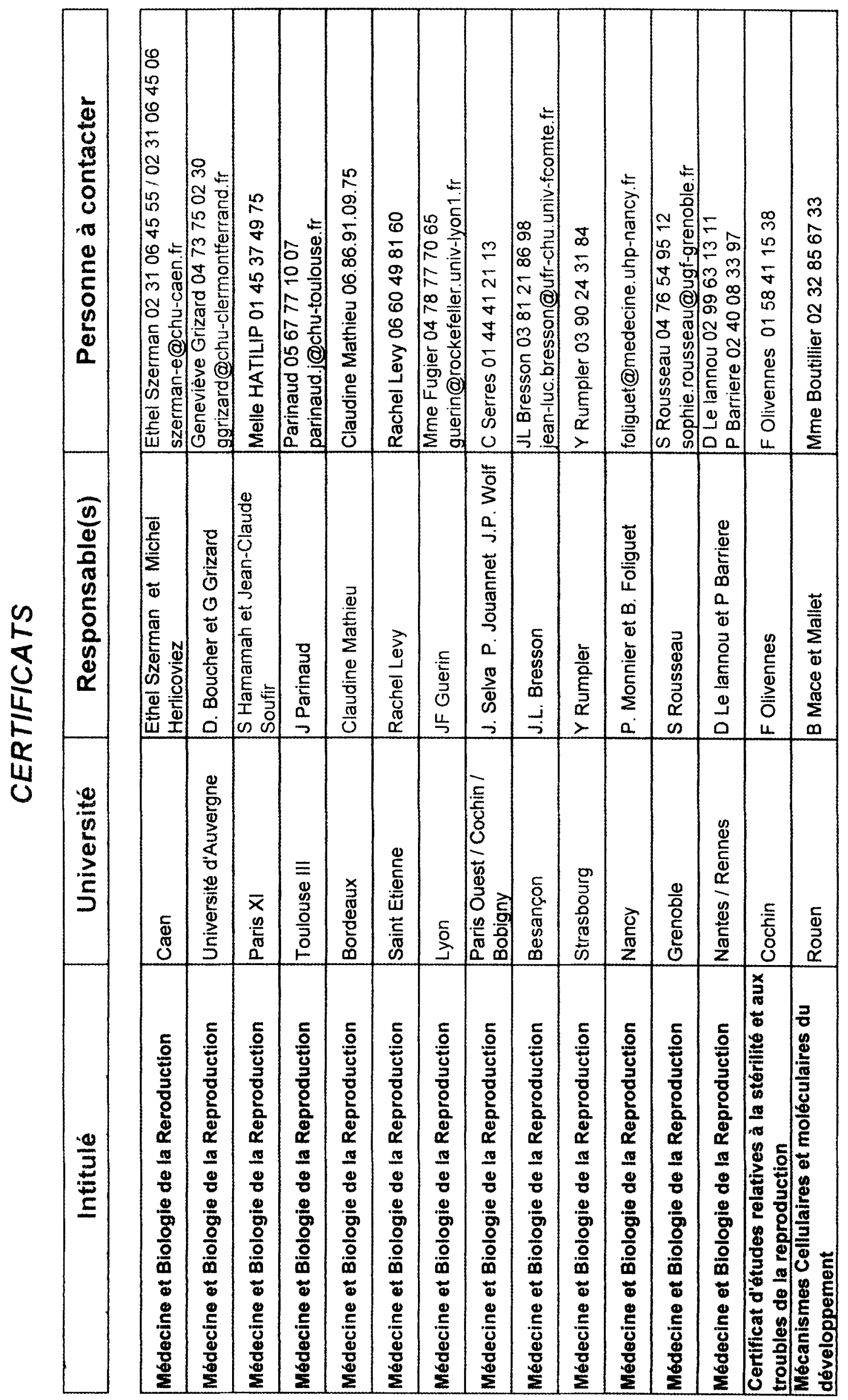




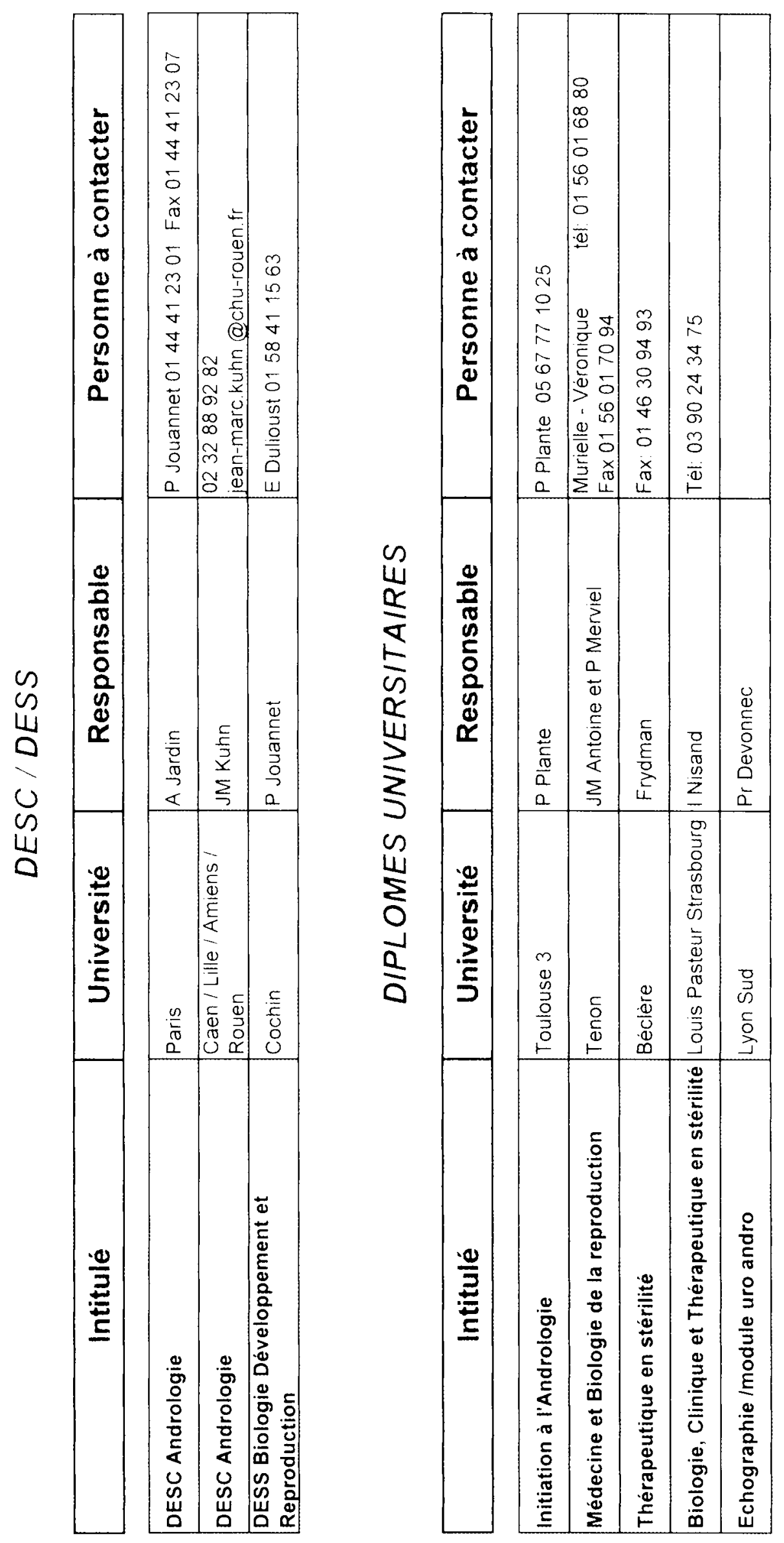




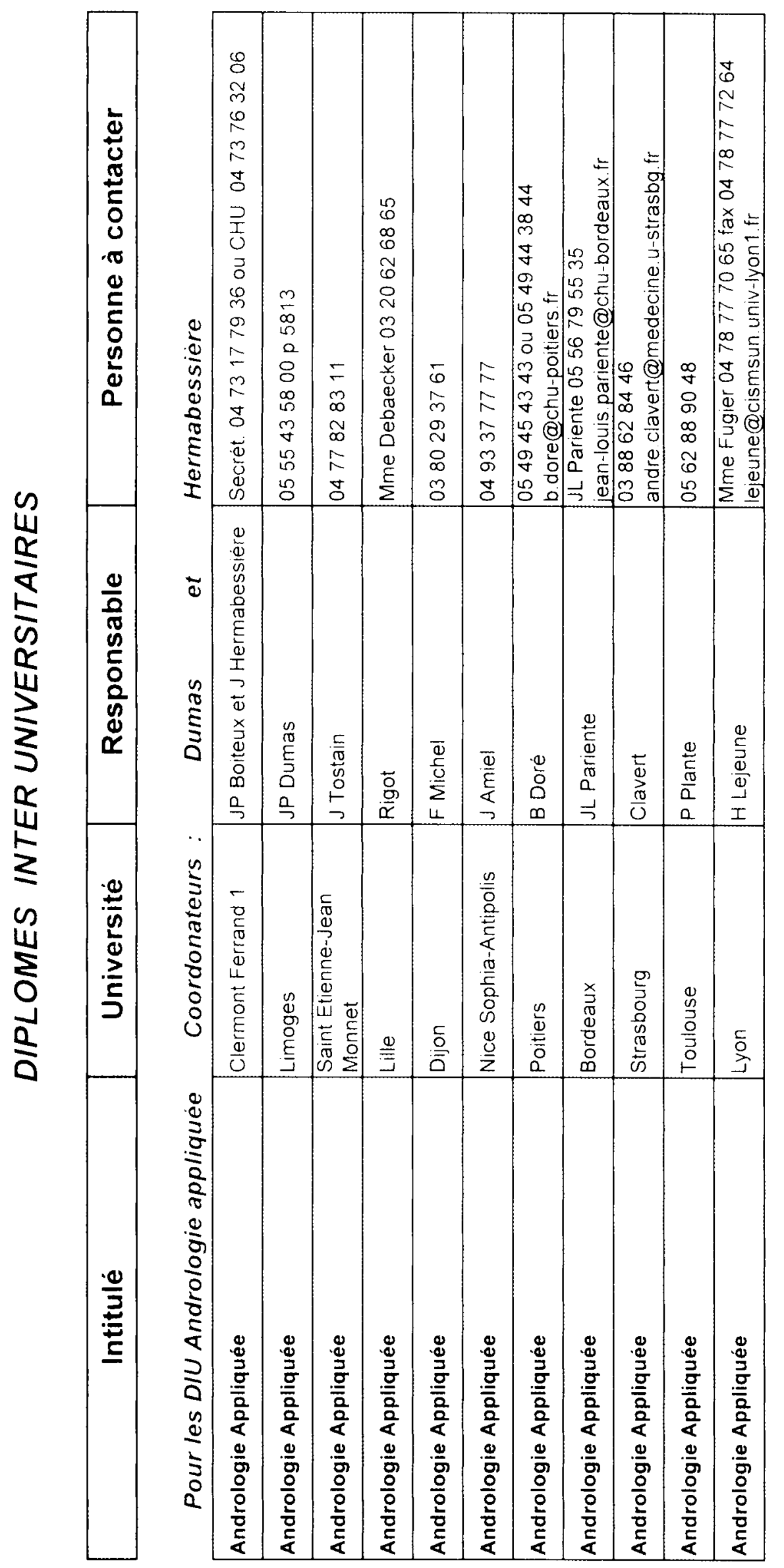




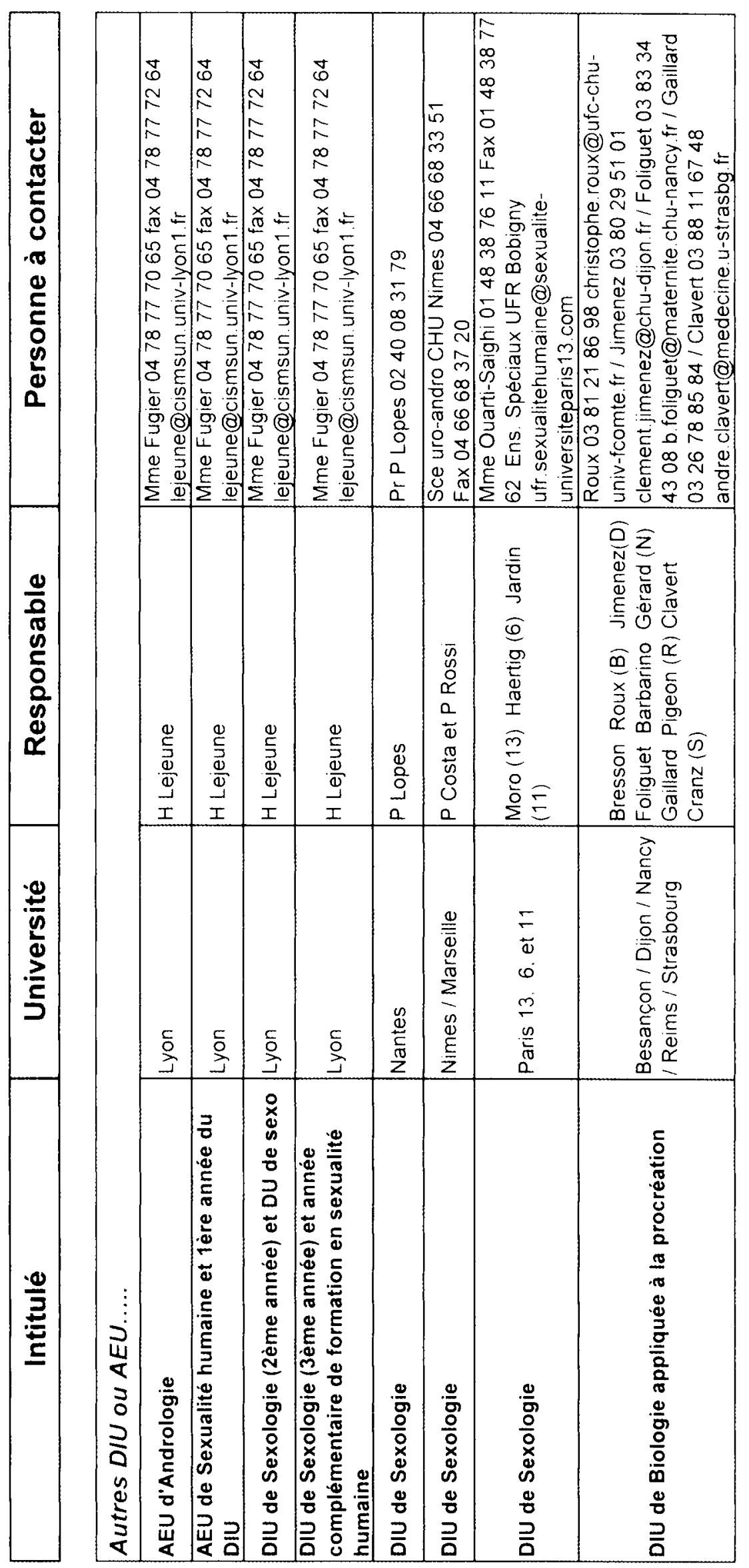




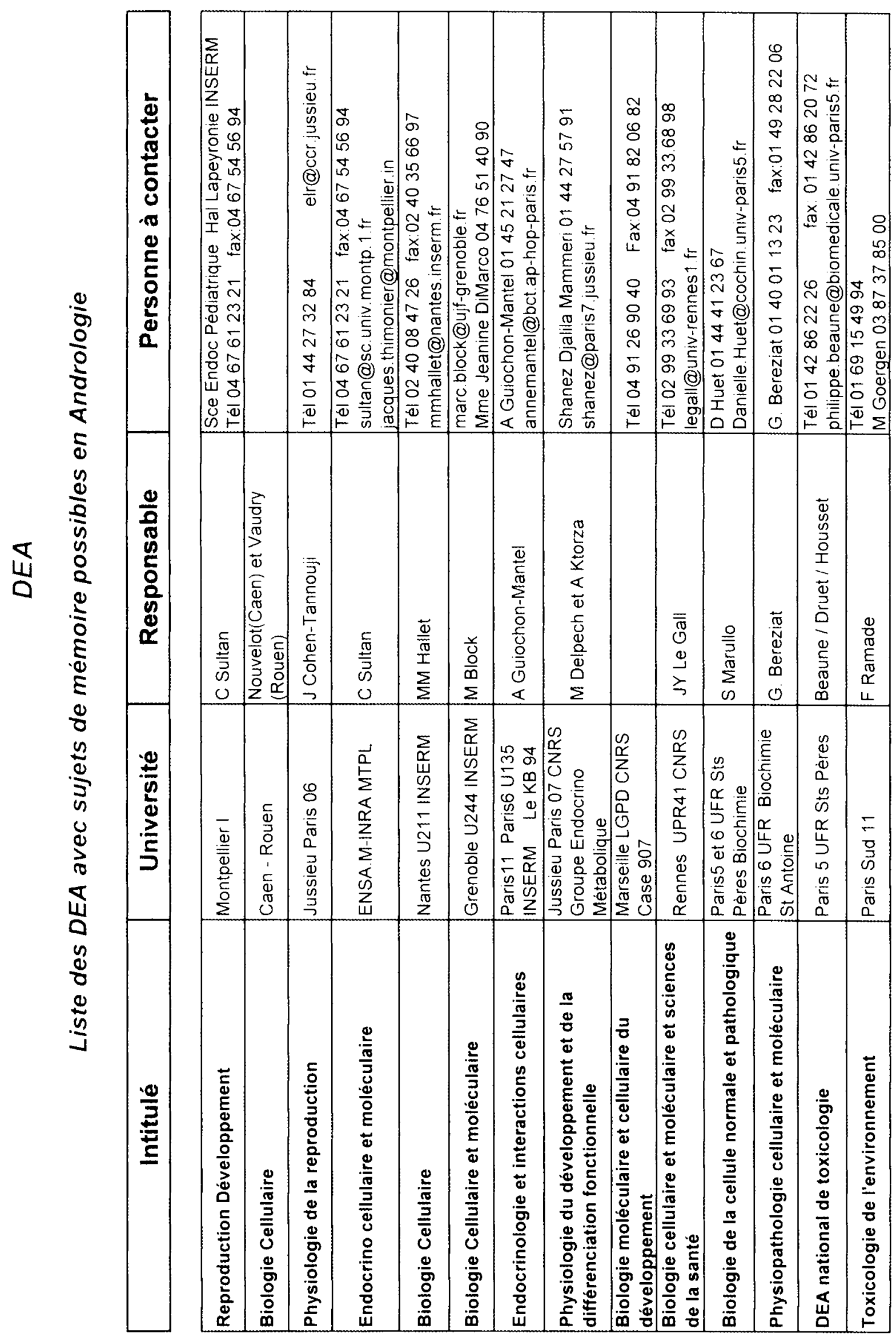




\begin{tabular}{|c|c|c|c|c|c|c|}
\hline 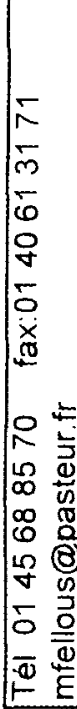 & 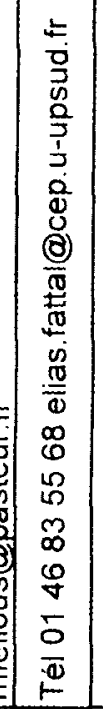 & 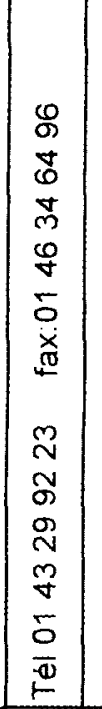 & 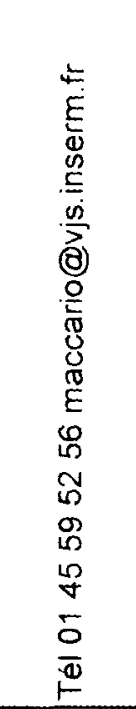 & & 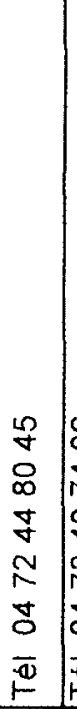 & 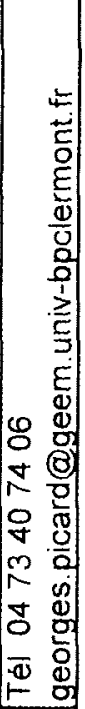 \\
\hline 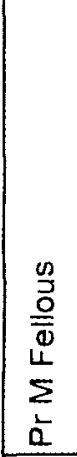 & 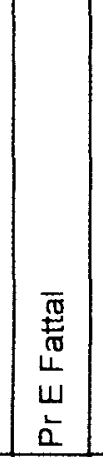 & & 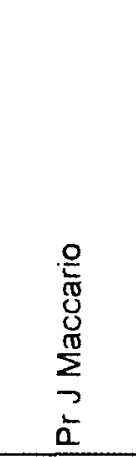 & \begin{tabular}{|c|} 
\\
0 \\
0 \\
0 \\
0 \\
0 \\
0
\end{tabular} & $\begin{array}{l}\text { 로 } \\
\text { a } \\
0 \\
\underline{2} \\
\end{array}$ & $\begin{array}{l}0 \\
\frac{0}{\pi} \\
\frac{0}{2} \\
0 \\
0 \\
0 \\
\end{array}$ \\
\hline 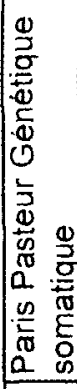 & 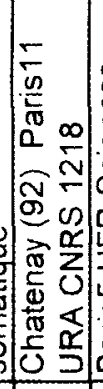 & 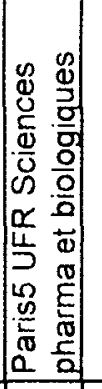 & 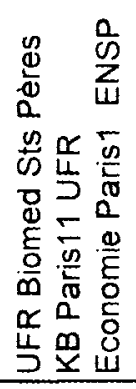 & 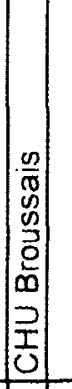 & 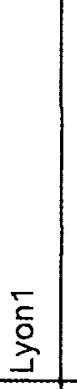 & 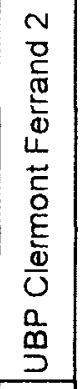 \\
\hline 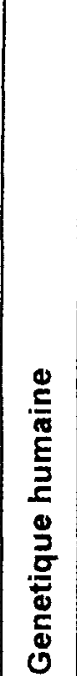 & 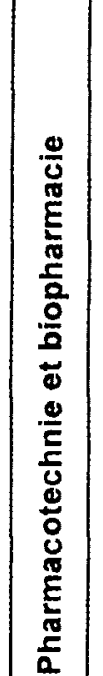 & 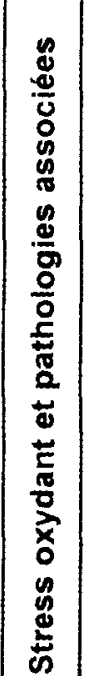 & 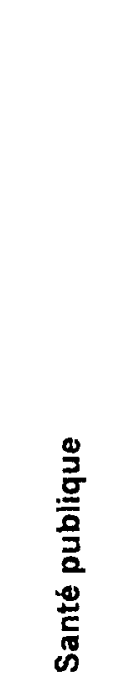 & 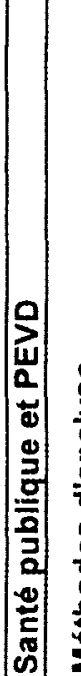 & 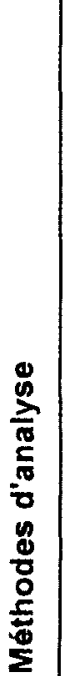 & 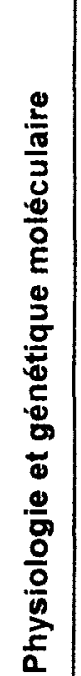 \\
\hline
\end{tabular}



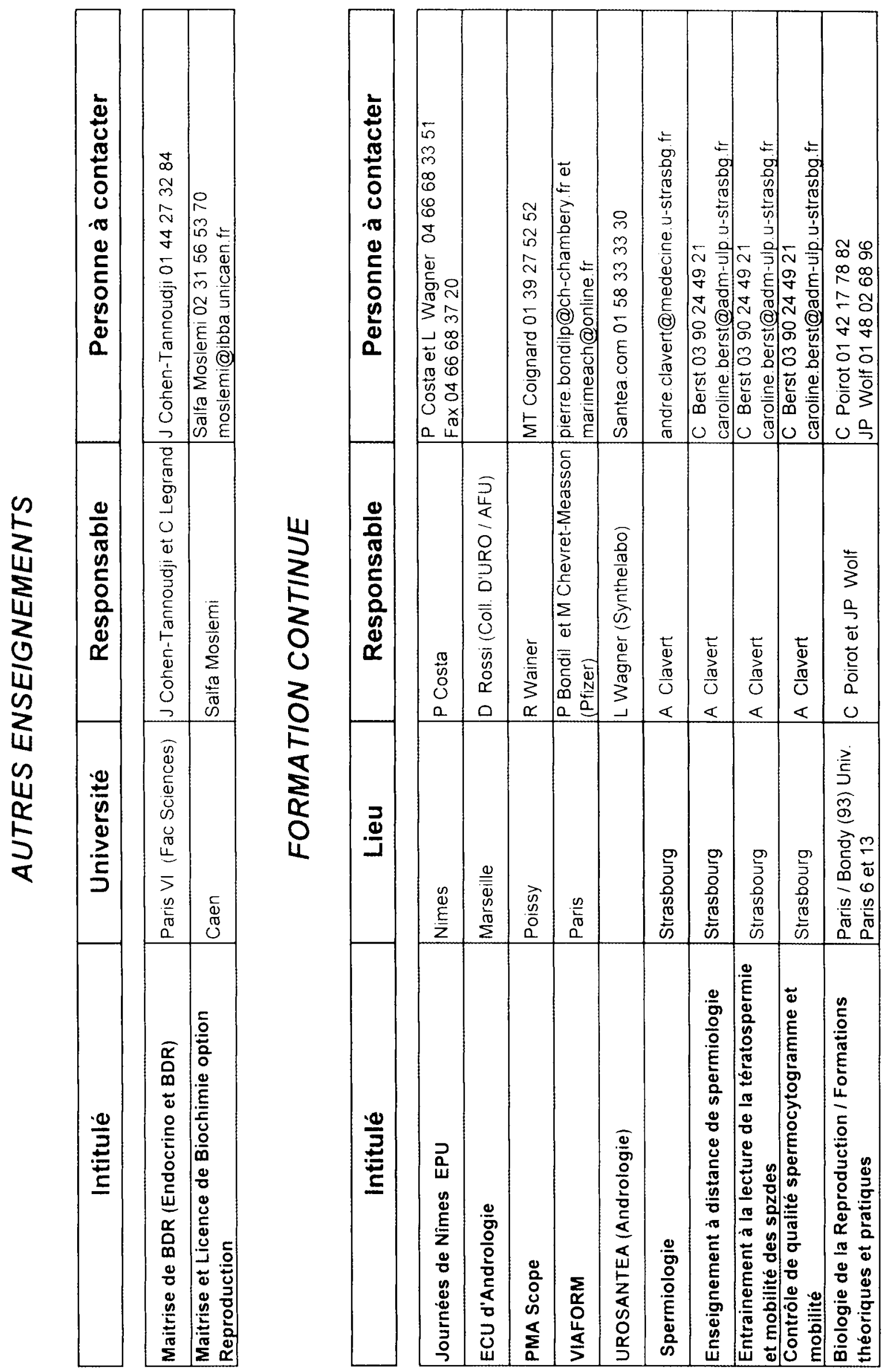